\title{
Relación entre nivel de adherencia al tratamiento y apoyo social en pacientes con falla cardiaca*
}

\author{
Relationship between Treatment Adherence Level and Social Support in Patients with Heart Failure Relação \\ entre nível de adesão ao tratamento e suporte social em pacientes com insuficiência cardíaca
}

Silvia Juliana Jaraba Suárez ${ }^{\mathrm{a}}$

Hospital Universitario San Ignacio, Colombia

DOI: https://doi.org/10.11144/Javeriana.ie23.rnat

dmunozacuna@unillanos.edu.co

ORCID: https://orcid.org/0000-0002-7486-7980

Recibido: 06 Abril 2021

Doraly Muñoz Acuña

Aceptado: 14 Octubre 2021

Universidad de los Llanos, Colombia

Publicado: 30 Diciembre 2021

Redalyc: http://www.redalyc.org/autor.oa?id=https://

www.redalyc.org/autor.oa?id=0000-0001-9062-2687

Margarita Maria Pomar Hoyos

Fundación Santa Fe de Bogotá, Colombia

ORCID: https://orcid.org/0000-0001-5858-0472

\section{Resumen:}

Introducción: La falla cardiaca (FC) es una de las enfermedades cardiovasculares con mayor índice de hospitalizaciones en la unidad de cuidado intensivo. En esta enfermedad es imperativo determinar aquellos factores asociados con el reingreso, como la adherencia al tratamiento y el apoyo social. Objetivo: determinar la relación entre la adherencia al tratamiento y el apoyo social de pacientes con falla cardiaca. Método: se realizó un estudio correlacional, con muestreo no probabilístico; el tamaño de la muestra fue de 64 pacientes con diagnóstico de FC. Para la medición del nivel de adherencia, se utilizó la escala para evaluación de comportamientos de adherencia con un alfa Cronbach de 0,72, para medir la percepción del apoyo social; se empleó la escala Medical Outcomes Study Social Supportsurvey (MOS) con una alfa de Cronbach de 0,94. Se realizó un análisis descriptivo y, para establecer la relación del nivel de adherencia con el apoyo social, se aplicó la prueba de independencia de distribuciones categóricas de Fisher. Resultados: Los participantes, en su mayoría, se encontraron adheridos al tratamiento (56,2\%); el índice global de apoyo social (media: 76,18 ) y sus dimensiones se encontraron en un rango medio. En cuanto a la asociación entre la adherencia al tratamiento, el apoyo social emocional (p: 0,006336) y el apoyo afectivo (p: 0,03025), esta fue moderada. Conclusiones: Existe asociación entre la adherencia y el apoyo social, por lo que es fundamental que el profesional de enfermería incluya la evaluación e intervención del apoyo social previo al alta del paciente, para optimizar los niveles de adherencia.

Palabras clave: apoyo social, insuficiencia cardiaca, cumplimiento y adherencia al tratamiento.

\section{Abstract:}

Introduction: Hearth Failure (HF) is one of the cardiovascular diseases with higher numbers of admissions in the ICU. In these diseases, it is a must to determine the factors associated with re-admission like the treatment adherence and social support. Objective: To determine the relationship between treatment adherence and social support in patients with HF. Methods: A correlational study with non-probabilistic sampling was conducted. The sample size was 64 patients diagnosed with HF. To measure the adherence level, the adherence behavior evaluation scale with a Cronbach's alpha of 0.72 was used. To measure the social support perception, the Medical Outcomes Study Social Supportsurvey (MOS) with a Cronbach's alpha of 0.94 was used. A descriptive analysis was carried out and in order to determine how the adherence level is related to the social support, the Fisher's test for categorical distributions was applied. Results: Most of the subjects showed a level of frequently an adhered to the treatment (56.2\%). The global index of social support was found with values in a mean range (mean: 76.18). The association between the treatment adherence, social support ( $\mathrm{p}: 0.006336$ ) and affective support ( $\mathrm{p}: 0.03025$ ) was moderate. Conclusions: There is an association between the treatment adherence and social support. Therefore, it is pivotal that the nurse include the evaluation and intervention of social support before the patient's discharge in order to optimize the adherence levels.

Keywords: social support, heart insufficiency, treatment compliance and adherence.

Notas de autor

\footnotetext{
a Autora de correspondencia:dmunozacuna@unillanos.edu.co
} 


\section{Resumo:}

Introdução: A insuAciência cardíaca (FC) é uma das doenças cardiovasculares com maior índice de internações na unidade de terapia intensiva. Nesta doença é imperativo determinar os fatores associados à readmissão, como a adesão ao tratamento e o suporte social. Objetivo: determinar a relação entre adesão a tratamento e o suporte social de pacientes com InsuAciência Cardíaca. Método: foi realizado um estudo correlacional, com amostragem não probabilística e tamanho da amostra de 64 pacientes com diagnóstico de IC. Para mensurar o nível de adesão, utilizou-se a Escala para avaliação de comportamentos de adesão com alfa Cronbach de 0,72; para medir a percepção do apoio social, usou-se a escala Medical Outcomes Study Social Supportsurvey (MOS) com alfa de Cronbach de 0,94. Realizou-se análise descritiva e, para estabelecer a relação entre o nível de adesão e o suporte social, foi aplicado o teste de independência de distribuições categóricas de Fisher. Resultados: a maioria dos participantes encontrava-se em nível de adesão frequente ao tratamento (56,2\%); o índice global de apoio social (média: 76,18) e suas dimensões se encontraram em um rango médio. Quanto à associação entre adesão ao tratamento, suporte social emocional (p: 0,006336) e suporte afetivo (p: 0,03025) foi moderada. Conclusões: Há associação entre adesão e suporte social, pelo que é fundamental que o proAssional de enfermagem inclua a avaliação e intervenção do apoio social antes da alta, para aperfeiçoar os níveis de adesão.

Palavras-chave: suporte social, insuAciência cardíaca, conformidade e adesão ao tratamento.

\section{Introducción}

La falla cardiaca (FC) es un síndrome clínico con compromiso funcional y estructural del corazón. Cuando avanza, este se caracteriza por síntomas como diAcultad respiratoria y fatigabilidad fácil, con signos como ingurgitación yugular, estertores pulmonares y edema en miembros inferiores (1); Según la American Heart Association (AHA), esta enfermedad presenta una alta prevalencia a nivel mundial, lo que genera un impacto negativo en la calidad de vida del paciente (2). En Colombia la mayor causa de mortalidad fueron las enfermedades del sistema circulatorio (3).

La adherencia al tratamiento, según (4), se deAne como "El grado en que el comportamiento de una persona - tomar el medicamento, seguir el régimen alimentario y ejecutar cambios del modo de vida - se corresponde con las recomendaciones acordadas de un prestador de asistencia sanitaria”. Así, en la medida que el paciente modiaque sus comportamientos, la adherencia se convertirte en un factor clave y condicionado en el mejoramiento de la calidad de vida del paciente con FC (5). No obstante, medir la adherencia al tratamiento en los pacientes con FC nos brinda información útil para el desarrollo de estrategias que conduzcan al paciente a una adaptación de comportamientos positivos y de autocuidado, para mejorar su salud y así disminuir los reingresos y los costos hospitalarios.

El contar con un adecuado apoyo social (AS), este se convierte en un generador de bienestar psicológico y físico en el paciente (6). En el AS el individuo consigue ayuda emocional, instrumental o económica; contar con un AS, primordialmente familiar, genera mejor control y afrontamiento de su enfermedad, ocasiona una menor demanda de atención en servicios de salud de mayor complejidad, disminuye la situación de estrés y origina un efecto protector directo y amortiguador, por lo que atenúa impactos adversos de la enfermedad crónica (7).

Mediante el respaldo de referentes teóricos, como la teoría de mediano rango de la enfermera Schaffer M., se recalca que el apoyo social es un aspecto facilitador para la promoción y prevención de problemas asociados con la cronicidad. Al mismo tiempo, se indica que, si se cuenta con redes sociales estresantes, con Áictivas o con un soporte ausente, esto afecta de forma negativa la recuperación del paciente (8).

En la actualidad nacional, se evidencia la ausencia de estudios correlacionales que permitan determinar la relación entre el nivel de adherencia al tratamiento farmacológico y no farmacológico y el apoyo social de los pacientes con falla cardiaca. Esto evidencia la necesidad de que el profesional de enfermería pueda generar un reconocimiento precoz del grado de adherencia y apoyo social con el que cuenta el paciente antes del alta institucional, y genere estrategias que los potencialicen. 


\section{Método}

Se realizó un estudio observacional correlacional, en una institución de III nivel. La muestra fue de 64 pacientes con diagnóstico de FC con clase funcional III o IV, con hemodinámicamente estable adecuada y capacidad cognitiva. El reclutamiento fue durante la estancia hospitalaria de los pacientes, y se hizo con el apoyo de la base de datos de la institución; luego, mediante una entrevista, quienes aceptaron, firmaron un consentimiento informado. Se realizó una prueba piloto para refinar la aplicación de los instrumentos, antes del reclutamiento de los pacientes.

Para la medición del nivel de adherencia, se utilizó el cuestionario "Evaluación de comportamientos de adherencia al tratamiento farmacológico y no farmacológico en los pacientes con falla cardiaca" diseñado por (9), validado en la población Colombiana, y un alfa Cronbach de 0,72; instrumento de 30 ítems y 10 dimensiones, con una escala Likert con cuatro opciones de respuesta, donde 1 corresponde a nunca y 4 a siempre, y permite clasificar el nivel de adherencia global, a través de los rangos: no adherente (30-55), poca adherencia (56-80), frecuentemente adherido (81-105), y adherido (106-120) y la adherencia en cada dimensión. El análisis psicométrico mostró un índice de fiabilidad de 0,7213.

Para medir la percepción del apoyo social, se utilizó la escala Medical Outcomes Study Social Supportsurvey (MOS) (10), validada en la población Colombiana, con un alfa de Cronbach de 0,94; el instrumento evalúa los siguientes componentes: a) red de apoyo social (amigos y familiares); b) apoyo social emocional/informacional: soporte emocional, orientación y consejos; c) apoyo instrumental: conducta o material de apoyo; d) interacción social positiva: disponibilidad de individuos con los que se pueda hacer cosas divertidas; y e) apoyo afectivo: expresiones de amor y afecto. El instrumento está constituido por 20 ítems, tiene una escala de respuesta tipo Likert donde 1 es nunca y 5 es siempre, cuenta con cuatro dimensiones con valores de mínimo, medio y máximo respectivamente: apoyo emocional $(8,24,40)$, ayuda material $(4,12,20)$, relaciones sociales de ocio y distracción $(4,12,20)$, apoyo afectivo $(3,9,15)$, y permite evaluar el índice global $(19,57,95)$. En cuanto a la capacidad cognitiva, esta fue revisada mediante la aplicación del test Minimental.

Los datos se consolidaron y se les realizó un análisis descriptivo para las variables dependiente (Adherencia al tratamiento) y Variables independientes (Apoyo social percibido). Para el análisis de las variables cuantitativas, se realizó el cálculo de medidas de tendencia central, de dispersión y medidas de posición; las variables cualitativas se hicieron mediante tablas de distribución porcentual y de frecuencia; y para establecer la relación del nivel de adherencia con el apoyo social, se aplicó la prueba de independencia de distribuciones categóricas de Fisher; y de acuerdo con los resultados de significancia estadística, se utilizó un estadístico de contingencia (Cramer V) para definir la fuerza de correlación entre el índice global de adherencia y los cuatro componentes del apoyo social. La información fue analizada en el software estadístico R versión 3.6.3.

En cuanto a los aspectos éticos, se realizó el diligenciamiento del consentimiento informado; también se tuvo en cuenta la normatividad para la investigación en seres Humanos, con las pautas éticas internacionales del (CIOMS) y la OMS, y a nivel nacional para investigación en salud, de la Resolución 8430 de 1993, igual que la declaración internacional de Helsinki. Está es una investigación "sin riesgo" al no realizarse ningún tipo de intervención en la población de estudio. De igual manera, se consideró que por el tipo de investigación y por los objetivos de la misma no causa ningún impacto ambiental.

\section{Resultados}

Frente a las características sociodemográficas, la muestra estuvo representada por personas entre los 37 años, como edad mínima, y 95 años, edad máxima, la media fue de 69,8 años y la desviación estándar (+- 12); el género de prevalencia fue de masculino $66 \%$ y femenino $34 \%$. Con relación al estado civil, se evidenció 
que el $45 \%$ eran casados y el 22\% viudos; la mayor proporción de pacientes contaba con una escolaridad primaria $67 \%$ y pertenecían al estrato 2 y 3 ; el $43 \%$ estaba pensionado, el $25 \%$ trabaja como independiente. Los pacientes contaban con un cuidador principal, para el $45 \%$ era la pareja, seguido de los hijos $33 \%$. Además, se identificó un tiempo menor de 10 años de diagnóstico, en donde la etiología isquémico y valvular fue la de mayor frecuencia. Respecto al número de medicamentos, la mayoría de los pacientes recibían más de 5 medicamentos, evidenciándose la polifarmacia en este grupo poblacional. El $90 \%$ ha presentado dos reingresos en el último año por descompensación de la enfermedad. El índice global de adherencia en la mayoría de los pacientes estaba en un nivel de frecuentemente adherido $56,2 \%$ y poco adherido $42,2 \%$, lo que evidencia la dificultad en el mantenimiento de un óptimo control de los comportamientos de adherencia (tabla 1).

TABLA 1

Medición global de la adherencia al tratamiento farmacológico y no farmacológico de los pacientes con diagnóstico de falla cardiaca hospitalizados

\begin{tabular}{|l|c|c|c|c|c|}
\hline $\begin{array}{l}\text { Rango de } \\
\text { adherencia }\end{array}$ & Frecuencia & $\begin{array}{c}\text { Porcentaj } \\
\text { e }\end{array}$ & $\begin{array}{c}\text { Rango de } \\
\text { adherencia }\end{array}$ & Frecuencia & $\begin{array}{c}\text { Porcentaj } \\
\text { e }\end{array}$ \\
\hline No adherente & 1 & 1,6 & $\begin{array}{c}\text { Frecuentemente } \\
\text { adherido }\end{array}$ & 36 & 56,2 \\
\hline $\begin{array}{l}\text { Poco } \\
\text { adherente }\end{array}$ & 27 & 42,2 & Adherido & 0 & 0 \\
\hline
\end{tabular}

Fuente: Elaboración Propia.

El instrumento permitió medir las dimensiones que componen la valoración de comportamientos de adherencia mediante una forma más específica.

Las dimensiones que evidenciaron poca adherencia fueron: 1) conocimiento del tratamiento farmacológico, 2) aceptación del diagnóstico dado por el profesional de la salud, 3) reconocimiento de signos y síntomas, 4) mantiene su peso dentro del rango recomendado y 5) conoce la dieta recomendada (ver tabla 2). Las dimensiones que evidenciaron en puntaje de frecuentemente adherido fueron: 1) confía en el profesional de la salud, 2) cuenta con información sobre la posibilidad de acceso a los servicios, 3) busca refuerzo externo para poder cumplir con los comportamientos de salud, 4) realiza ejercicio diario, según lo recomendado por el profesional de la salud, 5) estado de ánimo apropiado para la salud (ver tabla 2).

TABLA 2

Nivel de adherencia al tratamiento farmacológico y no farmacológico de los pacientes con diagnóstico de falla cardiaca hospitalizados

\begin{tabular}{|c|c|c|c|c|}
\hline Dimensiones & $\begin{array}{c}\text { Total, de } \\
\text { subindicadore } \\
\mathrm{s}\end{array}$ & $\begin{array}{l}\text { Rango } \\
\text { Escala } \\
\text { Likert }\end{array}$ & Interpretación & $\begin{array}{c}\begin{array}{c}\text { Promedio de } \\
\text { adherencia: } \\
\text { n64 }\end{array} \\
\end{array}$ \\
\hline $\begin{array}{l}\text { 1. confia en el } \\
\text { profesional de la } \\
\text { salud }\end{array}$ & $\begin{array}{l}1 \\
2\end{array}$ & $\begin{array}{l}2.0-3.5 \\
3.6-5.5 \\
5.6-7.0 \\
7.1-8.0\end{array}$ & $\begin{array}{l}\text { No adherente } \\
\text { Poca adherencia } \\
\text { Frecuentemente } \\
\text { adherido } \\
\text { Adherido }\end{array}$ & $\begin{array}{l}6,7(\mathrm{DE} \pm 1) \\
\text { Frecuentement } \\
\text { e adherido }\end{array}$ \\
\hline $\begin{array}{l}\text { 2. conocimiento } \\
\text { del tratamiento } \\
\text { farmacológico }\end{array}$ & $\begin{array}{l}3 \\
4 \\
5 \\
6\end{array}$ & $\begin{array}{l}4.0-7.0 \\
7.1-11.0 \\
11.1- \\
15.0 \\
15.1- \\
16.0 \\
\end{array}$ & $\begin{array}{l}\text { No adherente } \\
\text { Poca adherencia } \\
\text { Frecuentemente } \\
\text { adherido } \\
\text { Adherido }\end{array}$ & $\begin{array}{l}10,1(\mathrm{DE} \pm 2,5) \\
\text { Poca } \\
\text { adherencia }\end{array}$ \\
\hline $\begin{array}{l}\text { 3. Cuenta con } \\
\text { Información } \\
\text { sobre la } \\
\text { posibilidad de } \\
\text { acceso a los } \\
\text { servicios }\end{array}$ & $\begin{array}{l}7 \\
8 \\
9\end{array}$ & $\begin{array}{l}3.0-5.5 \\
5.6-8.0 \\
8.1-11.0 \\
11.1- \\
12.0\end{array}$ & $\begin{array}{l}\text { No adherente } \\
\text { Poca adherencia } \\
\text { Frecuentemente } \\
\text { adherido } \\
\text { Adherido }\end{array}$ & $\begin{array}{l}8,5(\mathrm{DE} \pm 1,8) \\
\text { Frecuentement } \\
\text { e adherido }\end{array}$ \\
\hline $\begin{array}{l}\text { 4. Aceptación } \\
\text { del diagnóstico } \\
\text { dado por el } \\
\text { profesional de la } \\
\text { salud }\end{array}$ & $\begin{array}{l}10 \\
11 \\
12 \\
13\end{array}$ & $\begin{array}{l}4.0-7.0 \\
7.1-11.0 \\
11.1- \\
15.0 \\
15.1- \\
16.0\end{array}$ & $\begin{array}{l}\text { No adherente } \\
\text { Poca adherencia } \\
\text { Frecuentemente } \\
\text { adherido } \\
\text { Adherido }\end{array}$ & $\begin{array}{l}10,8(\mathrm{DE} \pm 2,3) \\
\text { Poca } \\
\text { adherencia }\end{array}$ \\
\hline $\begin{array}{l}5 . \\
\text { Reconocimiento } \\
\text { de signos y } \\
\text { síntomas }\end{array}$ & $\begin{array}{l}14 \\
15 \\
16\end{array}$ & $\begin{array}{l}3.0-5.5 \\
5.6-8.0 \\
8.1-11.0 \\
11.1- \\
12.0\end{array}$ & $\begin{array}{l}\text { No adherente } \\
\text { Poca adherencia } \\
\text { Frecuentemente } \\
\text { adherido } \\
\text { Adherido }\end{array}$ & $\begin{array}{l}7,5(\mathrm{DE} \pm 1,7) \\
\text { Poca } \\
\text { adherencia }\end{array}$ \\
\hline
\end{tabular}




\begin{tabular}{|c|c|c|c|c|}
\hline $\begin{array}{l}\text { 6. Busca } \\
\text { refuerzo externo } \\
\text { para poder } \\
\text { cumplir con los } \\
\text { comportamiento } \\
\text { s de salud }\end{array}$ & $\begin{array}{l}17 \\
18\end{array}$ & $\begin{array}{l}2.0-3.5 \\
3.6-5.5 \\
5.6-7.0 \\
7.1-8.0\end{array}$ & $\begin{array}{l}\text { No adherente } \\
\text { Poca adherencia } \\
\text { Frecuentemente } \\
\text { adherido } \\
\text { Adherido }\end{array}$ & $\begin{array}{l}6(\mathrm{DE} \pm 1,5) \\
\text { Frecuentement } \\
\text { e adherido }\end{array}$ \\
\hline $\begin{array}{l}\text { 7. Mantiene su } \\
\text { peso dentro del } \\
\text { rango } \\
\text { recomendado }\end{array}$ & $\begin{array}{l}19 \\
20 \\
21 \\
22\end{array}$ & $\begin{array}{l}4.0-7.0 \\
7.1-11.0 \\
11.1- \\
15.0 \\
15.1- \\
16.0 \\
\end{array}$ & $\begin{array}{l}\text { No adherente } \\
\text { Poca adherencia } \\
\text { Frecuentemente } \\
\text { adherido } \\
\text { Adherido }\end{array}$ & $\begin{array}{l}8(\mathrm{DE} \pm 2,2) \\
\text { Poca } \\
\text { adherencia }\end{array}$ \\
\hline $\begin{array}{l}\text { 8. Conoce la } \\
\text { dieta } \\
\text { recomendada }\end{array}$ & $\begin{array}{l}23 \\
24\end{array}$ & $\begin{array}{l}2.0-3.5 \\
3.6-5.5 \\
5.6-7.0 \\
7.1-8.0\end{array}$ & $\begin{array}{l}\text { No adherente } \\
\text { Poca adherencia } \\
\text { Frecuentemente } \\
\text { adherido } \\
\text { Adherido }\end{array}$ & $\begin{array}{l}4,8(\mathrm{DE} \pm 1,3) \\
\text { Poca } \\
\text { adherencia }\end{array}$ \\
\hline $\begin{array}{l}\text { 9. Realiza } \\
\text { ejercicio diario, } \\
\text { según lo } \\
\text { recomendado } \\
\text { por el } \\
\text { profesional de la } \\
\text { salud }\end{array}$ & $\begin{array}{l}25 \\
26 \\
27\end{array}$ & $\begin{array}{l}3.0-5.5 \\
5.6-8.0 \\
8.1-11.0 \\
11.1- \\
12.0\end{array}$ & $\begin{array}{l}\text { No adherente } \\
\text { Poca adherencia } \\
\text { Frecuentemente } \\
\text { adherido } \\
\text { Adherido }\end{array}$ & $\begin{array}{l}8,4(\mathrm{DE} \pm 1,5) \\
\text { Frecuentement } \\
\text { e adherido }\end{array}$ \\
\hline $\begin{array}{l}\text { 10. Estado de } \\
\text { ánimo apropiado } \\
\text { para la salud }\end{array}$ & $\begin{array}{l}28 \\
29 \\
30\end{array}$ & $\begin{array}{l}3.0-5.5 \\
5.6-8.0 \\
8.1-11.0 \\
11.1- \\
12.0 \\
\end{array}$ & $\begin{array}{l}\text { No adherente } \\
\text { Poca adherencia } \\
\text { Frecuentemente } \\
\text { adherido } \\
\text { Adherido }\end{array}$ & $\begin{array}{l}10,4(\mathrm{DE} \pm 1,7) \\
\text { Frecuentement } \\
\text { e adherido }\end{array}$ \\
\hline
\end{tabular}

Fuente: Elaboración Propia.

El Índice global del apoyo social de los pacientes hospitalizados con diagnóstico de falla cardiaca se encontraba en un nivel medio de apoyo (media: 76,18- DE +-16,5). Igualmente, para las dimensiones que componen el apoyo social (emocional, material, relaciones, afectivo) se evidenció un nivel medio (ver tabla 3).

TABLA 3

Índice global de apoyo social pacientes con diagnóstico de falla cardiaca

\begin{tabular}{|l|c|c|c|}
\hline \multicolumn{1}{|c|}{ Dimensiones } & Media & Interpretación & Desviación estándar \\
\hline Apoyo emocional & 31,29 & Rango Medio & 7,61 \\
\hline Apoyo material & 17,18 & Rango Medio & 3,70 \\
\hline Apoyo en las relaciones & 15,68 & Rango Medio & 4,08 \\
\hline Apoyo afectivo & 12,01 & Rango Medio & 3,40 \\
\hline $\begin{array}{l}\text { Índice global de apoyo } \\
\text { social }\end{array}$ & 76,18 & Rango Medio & 16,52 \\
\hline
\end{tabular}

Fuente: Elaboración Propia.

Respecto a la relación entre el índice global apoyo social y la adherencia farmacológica y no farmacológica en los pacientes de falla cardiaca, quienes se encontraban frecuentemente adheridos $(56,2 \%)$ o poco adheridos $(42,2 \%)$ contaban un índice global de apoyo social medio, apoyo material, relaciones de ocio y distracción y apoyo afectivo.

Al utilizar la prueba exacta de Fisher, como prueba de significación estadística de distribuciones categóricas, se evidenció la relación entre la adherencia al tratamiento y el apoyo social. Allí se encontró una asociación del nivel de apoyo emocional (p: 0,006) y el nivel de apoyo afectivo (p: 0,030) con los niveles de adherencia al tratamiento.

Igualmente, el estadístico Cramer V permitió medir el tamaño del efecto entre las dimensiones de apoyo social y la adherencia al tratamiento, evidenciando una asociación moderada entre emocional $(0,32)$ y afectiva $(0,29)$ con la adherencia al tratamiento (ver tabla 4$)$.

TABLA 4

Descripción de la relación de las dimensiones del apoyo social con la adherencia al tratamiento

\begin{tabular}{|l|c|c|}
\hline \multicolumn{1}{|c|}{ Variables } & Valor $\mathbf{P}^{*}$ & $\begin{array}{c}\text { Fuerza de Correlación } \\
* \star\end{array}$ \\
\hline Adherencia vs. Apoyo emocional & P: 0,006 & 0,32 \\
\hline Adherencia vs. Apoyo Material & P: 0,114 & - \\
\hline $\begin{array}{l}\text { Adherencia vs. Apoyo en las } \\
\text { Relaciones }\end{array}$ & P: 0,235 & - \\
\hline Adherencia vs. Apoyo afectivo & P: 0,030 & 0,29 \\
\hline
\end{tabular}

* Prueba de Fischer ${ }^{* *}$

Prueba Cramer V

Fuente: Elaboración Propia 


\section{Discusión}

En Colombia, no se evidencian investigaciones que demuestren la correlación entre el apoyo social y la adherencia al tratamiento en los pacientes con falla cardiaca. Frente a las características sociodemográficas, la edad media fue de 69,8 años, la escolaridad primaria fue la predominante, junto con el estrato socioeconómico 2 y 3; así como una red de apoyo en la que prevalecen los cónyuges e hijos. En el estudio (11) se manifiesta que los adultos mayores son quienes presentan más comorbilidades, lo que conlleva a un deterioro cognitivo por el cual van a requerir mayor apoyo social para afrontar la enfermedad; además, observaron que poseer niveles educativos bajos puede afectar la accesibilidad a los servicios de salud.

Con relación al apoyo social, (12) evidencia que, al ser la familia nuclear la principal red de apoyo, esta ocasionara conductas de adherencia y de autocuidado en el paciente mejorando su calidad de vida. Frente a las variables clínicas, se encontró que los pacientes tenían una etiología de la enfermedad de origen isquémico, con una fracción de eyección intermedia, una clase funcional NYHA III - IV y recibían más de 5 medicamentos para el manejo de su patología. Lo anterior concuerda con (13), quienes indicaron en sus estudios que los pacientes con este tipo de etiología y fracción de eyección son más susceptibles a presentar descompensaciones hemodinámicas por la severidad en la disfunción ventricular, ocasionando reingresos hospitalarios y deteriorando la calidad de vida del paciente, aspecto que es evidente debido a que más del $90 \%$ de los participantes presentaron reingresos en el último año. (13) también indicó que la polifarmacia puede generar el aumento de eventos adversos relacionados con la administración inapropiada de medicamentos, lo que conlleva a interacciones farmacológicas desfavorables y facilita el incumplimiento en la toma.

En cuanto a los niveles globales de adherencia al tratamiento, se determinó que la mayoría de los pacientes tenían un nivel "frecuentemente adherido", seguido de "poca adherencia"; resultados que coinciden con lo encontrado por (14), quienes manifestaron que es imperativo mejorar el nivel de adherencia mediante el fortalecimiento de actividades que promuevan el autocuidado para mejorar la calidad de vida de estos pacientes. Se encontraron bajos niveles de adherencia frente al tratamiento farmacológico, la dieta, mantenimiento de peso y reconocimiento de signos y síntomas. Los resultados coinciden con lo expuesto por (15), quienes indicaron que estos resultados conllevan a mayor riesgo de requerir hospitalizaciones prolongadas y a largo plazo disminución de la supervivencia. Las dimensiones que puntuaron como "frecuentemente adheridas" fueron las de 1) confianza en el profesional de la salud, 2) accesibilidad a los servicios, 3) búsqueda de refuerzo y 4) estado de ánimo, lo cual, según (16), indica que este tipo de hallazgos es considerado como un predictor de adherencia, que genera un bienestar emocional y aumenta su autoeficacia.

Los pacientes contaban con un índice global de apoyo social percibido en un nivel medio, donde la familia es la principal fuente de apoyo. Esto se correlaciona con lo encontrado por (17), quienes resaltan que el apoyo de la familia mejora la autoestima y fomenta los pensamientos positivos; además de convertirse en un aspecto beneficioso para la salud. La mayoría de los pacientes que se encontraban frecuentemente adheridos, $56,2 \%$, y poco adheridos, $42,2 \%$, presentaron un índice global de apoyo social medio, resultado poco favorecedor debido a que, como lo menciono (18), tener niveles de apoyo social en rangos bajos o medios puede desencadenar trastornos afectivos como lo es la depresión.

Se encontró que, dentro del apoyo social las dimensiones de apoyo emocional (p: 0,006336) y de apoyo afectivo ( $\mathrm{p}:$ 0,03025) presentaron una asociación moderada, aspecto favorecedor en el afrontamiento de la enfermedad, pues lleva al paciente a tomar mejores decisiones sobre su condición clínica. En la investigación de (19) se mostró una asociación positiva entre el apoyo afectivo y la adherencia, las cuales generaron conductas que facilitaron el mantenimiento de la salud. La dimensión emocional, que permite mostrar empatía y confianza, y la dimensión afectiva, que se entiende como las expresiones de amor ofrecidas por parte de su grupo más cercano (10), facilitan a los pacientes el afrontamiento de su enfermedad, permitiéndoles conocer las fases de adaptación que se producen en la enfermedad crónica. Los resultados de esta investigación revelaron que es imperativo identificar que el apoyo social es un componente favorecedor para que el paciente 
logre la adherencia al tratamiento, y se hace indispensable que el profesional de enfermería sea capaz de reconocer, durante la hospitalización y el egreso, el grado de adherencia del paciente, con el an de tener una visión global del tipo de apoyo con que cuenta el paciente, para poder potencializarlo; de esta manera, se pueden aAanzar conductas adherentes en el paciente.

\section{Limitaciones}

Una limitación del estudio es el tamaño muestral. Se recomienda en próximas investigaciones incrementar el tamaño de la muestra y realizar otras pruebas estadísticas que permitan examinar más la posible asociación del apoyo social con la adherencia.

\section{Conclusiones}

Las variables sociodemográAcas y clínicas: edad avanzada, género masculino, escolaridad primaria, polifarmacia y reingresos hospitalarios, podrían in f́uir en el nivel de adherencia.

La asociación entre el apoyo social y la adherencia al tratamiento farmacológico y no farmacológico en los pacientes que participaron de esta investigación demostró que contar con un apoyo social afectivo y emocional son determinantes para mejorar los niveles de adherencia al tratamiento; esto repercute en la calidad de vida no solo del paciente, sino de su familia.

Se demostró una asociación moderada entre la dimensión emocional y afectiva del apoyo social con la adherencia al tratamiento; es esencial que el profesional de enfermería comprenda las dimensiones del apoyo social con que cuenta el paciente, con el an de lograr optimizarlo para favorecer al mantenimiento de conductas de autocuidado, incluyendo una evaluación e intervención del apoyo social previamente al alta hospitalaria, que logre potencializar los niveles de adherencia.

\section{Referencias}

1. Gómez-Mesa JE, Saldarriaga C, Jurado AM, Mariño A, Rivera A, Herrera Á, et al. Consenso colombiano de falla cardíaca avanzada: capítulo de falla cardíaca, trasplante cardíaco e hipertensión pulmonar de la sociedad colombiana de cardiología y cirugía cardiovascular. Rev Colomb Cardiol. 2019;26(S2):3-24.https://doi.org/10 .1016/j.rccar.2019.06.001

2. Benjamin EJ, Muntner P, Alonso A, Bittencourt MS, Callaway CW, Carson AP, et al. Heart disease and stroke statistics 2019. Clinical Statements and guidelines. Circulation. 2019;139(10):e56-e528. https://doi.org/10.11 61/CIR.0000000000000659

3. Avella-Tolosa AJ. Análisis De Situación De Salud (ASIS). Bogotá: Ministerio de Salud y Protección social; 2019 mayo. 1.

4. Organización Mundial de la Salud. Adherencia a los tratamientos a largo plazo. Pruebas para la acción. Ginebra: Organización Mundial de la Salud; 2004.

5. Viñas-Vera C, García-Parra AM, Morales-Gil IM. Género y efectividad de la metodología enfermera en pacientes con insuAciencia cardiaca. Aquichan. 2016;16(3):313-327. https://doi.org/10.5294/aqui.2016.16.3.4

6. Cuadra-Peralta A, Medina-Cáceres EF, Salazar-Guerrero KJ. Relación de bienestar psicológico, apoyo social, estado de salud física y mental con calidad de vida en adultos mayores de la ciudad de Arica. Límite. 2016;35(11):56-67.

7. Marín-Bustamante D, Sanhueza-Alvarado O. Apoyo Social: Uso del Concepto en Enfermería. Rev Horiz Enferm. 2016;27(2):32-40. https://doi.org/10.7764/Horiz_Enferm.27.2.41

8. Peterson SJ, Bredow TS. Middle range theories: application to nursing research. 3ed. Filadelaa: Wolters Kluwer Health; 2013. Cap. IV, Social Support Marjorie A. Schaffer; p. 108-112. 
9. Achury-Saldaña DM, Sepúlveda-Carrillo GJ, Rodríguez-Colmenares SM, Giraldo IC. Validez y confiabilidad de un instrumento evaluativo de adherencia en pacientes con falla cardiaca. Enferm Global. 2012;11(26):1-9. https:/ /dx.doi.org/10.4321/S1695-61412012000200001

10. Londoño-Arredondo NE, Rogers H, Castilla-Tanga JF, Posada-Gómez SR, Ochoa-Arizal NL, Jaramillo-Pérez MA, et al. Validación en Colombia del cuestionario MOS de apoyo social. Int. J. Psychol. Res. 2012;5(1):142-150. http://mvint.usbmed.edu.co:8002/ojs/index.php/web

11. Sánchez SV, Olmos-Jiménez R, Ramírez-Roig C, García-Sánchez MJ, Valderrey-Pulido M. Adherencia al tratamiento en pacientes mayores de 65 años que sufren reingresos precoces. Farm Hospit. 2017;42(4):147-151. https://doi.org/10.15446/revfacmed.v64n4.52217

12. Arcos-Medina, L. C., Méndez-Toro, A., Rojas-Ruiz, I. T., Torres, S., \& Tabares, S. C. Caracterización clínico epidemiológica de pacientes hospitalizados con diagnóstico de falla cardiaca descompensada con fracción de eyección reducida del Hospital Militar Central. Acta médica colombiana. 2020;45(1). https://doi.org/10.361 04/amc.2020.1233

13. Muñoz-Mejía OA, Sierra-Vargas EC, Zapata-Cárdenas A, Isaza-Montoya M, Muñoz-Cifuentes MA, SánchezEchavarría JD. Caracterización sociodemográfica y clínica de una población con falla cardíaca aguda: cohorte MED-ICA. 2018;25(3):200-208. https://doi.org/10.1016/j.rccar.2017.12.021

14. Rojas-Sánchez LZ, Echeverría-Correa LE, Camargo-Figueroa FA. Adherencia al tratamiento farmacológico y no farmacológico en pacientes con falla cardiaca. Rev Elect Trim Enferm. 2014;36:1-14. https://doi.org/10.6018 /eglobal.13.4.178311.

15. Chang LL, Xu H, DeVore AD, Matsouaka RA, Yancy CW, Fonarow GC, Allen LA, Hernandez AF. Timing of Postdischarge Follow-Up and Medication Adherence Among Patients with Heart Failure. JAHA. 2018;7(7). h ttps://doi.org/10.1161/JAHA.117.007998

16. Cancino M, Rehbein-Felmer L, Ortiz MS. Funcionamiento cognitivo en adultos mayores: rol de la reserva cognitiva, apoyo social y depresión. Revista médica de Chile. 2018;146(3):315-322. http://dx.doi.org/10.4067 /s0034-98872018000300315

17. Molano Barrera D, González Consuegra R. Factores deteriorantes de la calidad de vida en insuficiencia cardiaca: revisión integrativa. Duazary. 2021;18(1):86-98. https://doi.org/10.21676/2389783X.3889

18. Gottlieb BH. Support Interventions: a typology and agenda for research. Handbook of personal relationships. Nueva York: Wiley and Sons; 1988.

19. Cené CW, Haymore, LB, Dolan-Soto D, Lin FC, Pignone M, Dewalt, DA, Wu JR, Jones CD, Corbie-Smith G. Self-care confidence mediates the relationship between perceived social support and self-care maintenance in adults with heart failure. J. Card. Fail. 2013;19(3):202-210. https://doi.org/10.1016/j.cardfail.2013.01.009

\section{Notas}

* Artículo original de investigación.

\section{Licencia Creative Commons CC BY 4.0}

Conflicto de intereses: Los autores declaran no tener conflicto de interés.

Financiación: Ninguna.

Cómo citar este artículo: Jaraba Suárez S. J., Muñoz Acuña D., Pomar Hoyos M. M. Relación entre nivel de adherencia al tratamiento y apoyo social en pacientes con falla cardiaca. Investigación en Enfermería, Imagen y Desarrollo. 2021;23. https://doi.org/10.11144/Javeriana.ie23.rnat 